$9-1-2009$

\title{
Assessment of Mixing in Passive Microchannels with Fractal Surface Patterning
}

\author{
Petru S. Fodor \\ Cleveland State University, p.fodor@csuohio.edu \\ M. Itomlenskis \\ Miron Kaufman \\ Cleveland State University, m.kaufman@csuohio.edu
}

Follow this and additional works at: https://engagedscholarship.csuohio.edu/sciphysics_facpub

Part of the Physics Commons

How does access to this work benefit you? Let us know!

\section{Publisher's Statement}

Copyright 2009 EDP Sciences. The original publication is available at http://www.epjap.org/ action/displayAbstract?fromPage=online\&aid=8010156.

\section{Original Citation}

Fodor, Petru S., M. Itomlenskis, and Miron Kaufman. "Assessment of Mixing in Passive Microchannels with Fractal Surface Patterning." European Physical Journal: Applied Physics 47 (2009): 31301.

\section{Repository Citation}

Fodor, Petru S.; Itomlenskis, M.; and Kaufman, Miron, "Assessment of Mixing in Passive Microchannels with Fractal Surface Patterning" (2009). Physics Faculty Publications. 114.

https://engagedscholarship.csuohio.edu/sciphysics_facpub/114

This Article is brought to you for free and open access by the Physics Department at EngagedScholarship@CSU. It has been accepted for inclusion in Physics Faculty Publications by an authorized administrator of EngagedScholarship@CSU. For more information, please contact library.es@csuohio.edu. 


\section{EPJ $A P$}

\section{Applied Physics}

Eur. Phys. J. Appl. Phys. 47, 31301 (2009)

DOI: $10.1051 /$ epjap/2009108

Assessment of mixing in passive microchannels with fractal surface patterning

P.S. Fodor, M. Itomlenskis and M. Kaufman

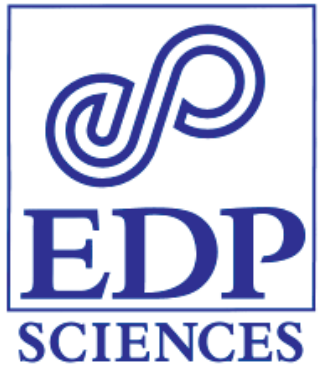

The title "The European Physical Journal" is a joint property of EDP Sciences, Società Italiana di Fisica (SIF) and Springer 


\title{
Assessment of mixing in passive microchannels with fractal surface patterning
}

\author{
P.S. Fodor ${ }^{\mathrm{a}}$, M. Itomlenskis, and M. Kaufman \\ Physics Department, Cleveland State University, Cleveland, OH 44115, USA
}

Received: 18 February 2009 / Accepted: 17 April 2009

Published online: 12 June 2009 - (C) EDP Sciences

\begin{abstract}
We explore numerically the feasibility of enhancing the mixing capability of microchannels by employing the Weierstrass fractal function to generate a pattern of V-shaped ridges on the channel floor. Motivated by experimental limitations such as the finite resolution $(\sim 10 \mu \mathrm{m})$ associated with rapid prototyping through soft lithography techniques, we study the influence on the quality of mixing of having finite width ridges. The mixing capability of the designs studied is evaluated using an entropic measure and the designs are optimized with respect to: the distances between the ridges and the position range of their tip along the width of the channels. The results are evaluated with respect to the benchmarks established by the very successful staggered herring bone (SHB) design. We find that the use of a non periodic protocol to generate the geometry of the bottom surface of the microchannels can lead to consistently larger entropic mixing indices than in cyclic structures. Furthermore, since the optimization curves (mixing index vs. geometric parameters) are broader at the maximum for fractal microchannels than for their SHB counterparts, the microchannel designs using the Weierstrass fractal function are less sensitive to experimental uncertainties.
\end{abstract}

PACS. $47.85 .1 \mathrm{k}$ Mixing enhancement $-47.53 .+\mathrm{n}$ Fractals in fluid dynamics - 47.61.Ne Micromixing

\section{Introduction}

Recent years have seen a tremendous increase in the level of research efforts targeted towards the use of microfluidic devices $[1,2]$ in medicine, biology, and chemistry. The applications of such systems range from chemical synthesis [3] and analysis [4], to enabling tools for biotechnology, such as DNA or protein analysis [5] and drug discovery [6]. The benefits of replacing "macro" fluid handling systems include the reduction in the consumption of samples and reagents; the enhanced performance in the reactions' speed due to increased surface to volume ratios; the ability to implement high throughput parallel sample processing [7] and the increased portability associated with the integration of various analytical processes within areas of the order of square centimeters $[8,9]$.

For drug delivery applications the microfluidic systems must efficiently mix different fluid components. However, in microchannels the Reynolds number is small $(<100)$, while the Peclet number is large $(>100)$ [10]. Thus the fluid flow in a pressure driven systems is typically laminar and the diffusion processes are slow compared to convection. Hence the design of microfluidic systems that are efficient mixers is challenging.

Broadly, the strategies employed to achieve efficient mixing of two or more fluid components within a microsys-

\footnotetext{
a e-mail: p.fodor@csuohio.edu
}

tem are classified as active and passive strategies. Active strategies rely on external sources of energy such as electrokinetic phenomena [11], magnetic forces [12], electroosmotic flows [13] or ultrasonic effects [14] to induce mixing. While these designs have demonstrated good mixing capabilities, this comes at the expense of increased complexity during the fabrication process which consequently precludes rapid prototyping during the optimization process. Also, the use of control signals, such as large electric fields might require strategies for heat management at the microfluidic chip level. On the other hand, passive micromixers, rely only on the geometry of the channels to perturb the laminar flow of the fluid and promote mixing. Thus, since they do not require interfacing with mechanical or electrical forces, they have the potential of reducing the manufacturing overhead and eliminating the challenges of interfacing with active control systems. Some passive mixers rely on the baker's transformation, in which the fluid streams are systematically split and recombined to geometrically increase the surface area between the fluid components. While this strategy has already been implemented [15-17] it extensively employs complicated 3D geometries which require multiple lithography steps. Other passive mixers have the shape of spirals $[18,19]$, where nonaxial flows (Dean flows) arise as a result of the centrifugal forces experienced by the fluids as they travel along curved trajectories. These flows are characterized by the presence 
of two counter-rotating vortices located below and above the symmetry plane of the channel.

In their pioneering work, Stroock et al. [20] have demonstrated that surface patterning of channels also provides a practical method to induce transversal flows across the channel cross section and thus promote mixing. In straight channels, slanted grooves present an anisotropic resistance profile to the fluid flow allowing for the axial pressure gradient between the inlet and outlet of the channels to drive transversal components of the flow. One of the designs investigated by Strook et al. [20], the staggered herring bone (SHB), which uses asymmetric $\mathrm{V}$-shaped grooves to produce counter-rotating transversal flows, has achieved two fluid components mixing within channel length scales of the order of centimetres making it relevant for lab-on-a-chip applications. Given the relatively straightforward fabrication that involves only a few lithographic steps and replica molding [8], several experimental studies have been aimed at improving the performance of this mixer by patterning structures on both the top and the bottom of the channel [21] or by adding oblique grooves on the side walls [22]. Moreover the geometrical simplicity of these designs makes them ideally suitable for computational fluid dynamics modelling through finite element analysis [23,24] and lattice Boltzmann approaches [25] and even simple analytical analysis [26]. Some of these studies have focused on determining the geometrical parameters that can affect the mixing performance, such as the groove depth to channel height ratio [27], the number of grooves per half cycle [25], the width of the grooves [28], the ratio between the width of the grooves with respect to the neighbouring ridges [29], or the ratio of the long arm to the short arm of the grooves [30].

A recent numerical investigation by Camesasca et al. [31] suggests that the quality of mixing can be further improved by replacing the periodic structure of ridges with a non-periodic one. In this approach the locations of the apexes are generated using the Weierstrass fractal function. In this study, we expand on the idea of using fractal surface patterning to enhance the chaotic character of the fluid flow to which the mixing in SHB channels is attributed [20]. We perform a computational optimization to identify the optimum ridge spacing and position range of the ridge apex. The performance of the proposed designs is compared with that of SHB designs with similar geometrical parameters, in order to asses the potential of these more complex systems to promote increased mixing of two fluid components.

\section{Microchannel geometry generation}

All the channels considered in this study have a length of $5000 \mu \mathrm{m}$, a width of $200 \mu \mathrm{m}$ and a height of $150 \mu \mathrm{m}$. The ratio between the ridge height and the height of the channel is fixed at 0.25 , the optimal ratio found for grooved micromixers [27]. As in the case of the SHB, a set of V-shaped ridge structures equally distributed along the channel bottom is employed. However, while in the case of the SHB

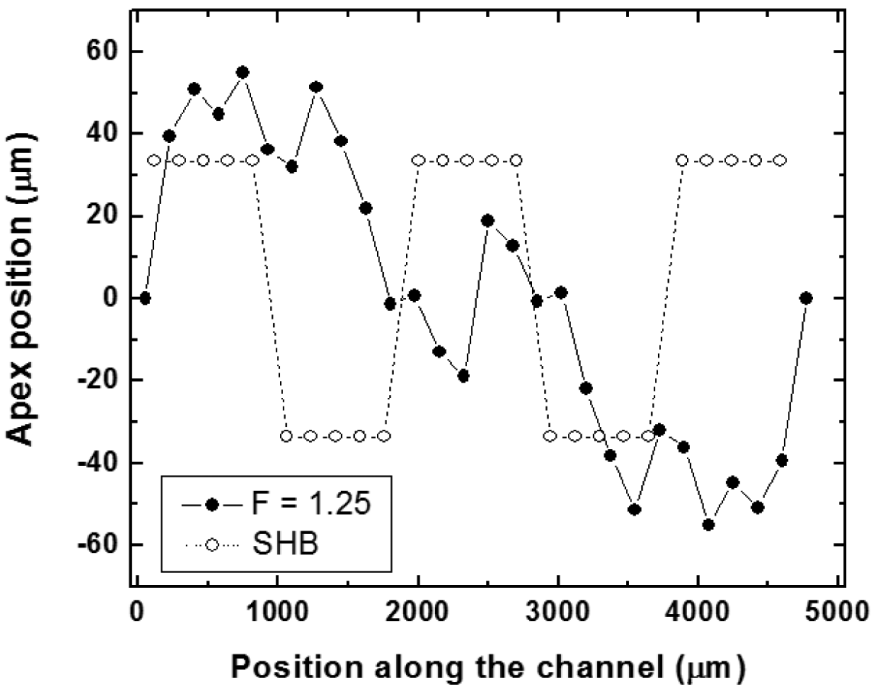

Fig. 1. Ridge apex $y$-coordinates as a function of the position along the channel length, for a SHB design and for a fractal dimension $F=1.25$ geometry, respectively. For both geometries the spacing between the ridges is $125 \mu \mathrm{m}$.

the apex position periodically alternates between two positions, corresponding to the short arm/long arm optimal ratio of $2 / 3$ [30], our strategy involves using a Weierstrass function to generate non-periodic sets of $y$-coordinates for the ridge tips (Fig. 1). The Weierstrass function used in this study is defined as:

$$
W(x)=\sum_{n=0}^{\infty} \frac{\sin \left(2^{n} x\right)}{2^{n(2-F)}}
$$

where $F$ is its fractal dimension and $x$ is the coordinate along the channel length.

For each predetermined ridge spacing, the domain of one period of the Weierstrass function, is evaluated at a number of points equal with $(5000 \mu \mathrm{m}) /[$ ridge width $(=50 \mu \mathrm{m})+$ spacing $(\mu \mathrm{m})]$ distributed at equi-sized intervals. Subsequently, a stretching transformation is applied to the set to distribute the apex points across the width of the channel. As described later, the performance of the mixer is sensitive to the apex position range allowed during the stretching transformation. For example for the design exemplified in Figure 1 the allowed apex range is $\pm 60 \mu \mathrm{m}$, rather than the full $200 \mu \mathrm{m}$ width of the channel.

Following the determination of the coordinates of the ridges' apexes along the width and length of the channels, the $\mathrm{V}$-shaped ridges are completed through a procedure similar with the SHB designs in which the ridges make a $45^{\circ}$ angle with the channel axis (Fig. 2b). This procedure is different from the one employed by Camesasca et al. [31] in their exploratory study of the effect of fractal surface patterning on mixing effectiveness in microchannels. In that case the ends of the arms of each V-ridge are aligned with the apex of the next ridge. The introduction of the new protocol for generating the geometry of the ridges was motivated by the restrictions imposed by the resolution capacity of rapid prototyping through 

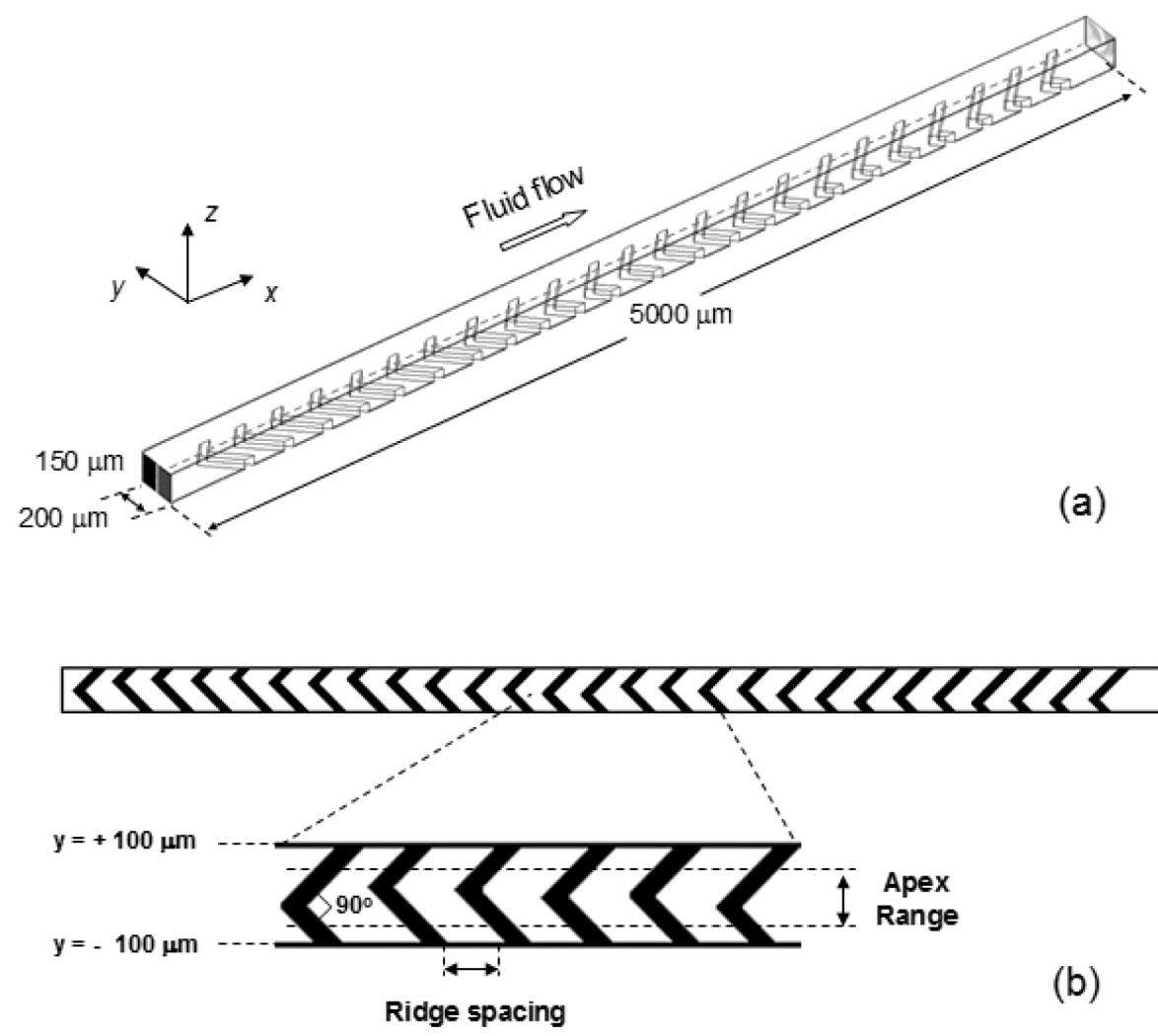

Fig. 2. (a) Typical 3D geometries for the rectangular microchannels with patterned bottom surface. (b) Top view of the ridge design for a structure based on the Weierstrass function with the fractal dimension $F=1.25$ (for the case illustrated the ridge spacing is $125 \mu \mathrm{m}$ ). The apex range is defined as the domain of ridge tips $y$-coordinates allowed during the stretching transformation.

soft lithography $(\sim 10 \mu \mathrm{m})$ techniques, which are becoming popular experimental approaches for implementing these designs.

To explore the consistency of this protocol in creating surface patterns capable to induce efficient mixing, several designs with different fractal dimensions $F=1.25,1.5$, and 1.75 have been analyzed.

\section{Numerical procedure and mixing analysis}

For all the simulations described in this work the finite element analysis package COMSOL Multiphysics and its Chemical Engineering Module were used. The flow fields for each channel are obtained by solving the NavierStokes equations of motion for an incompressible Newtonian fluid:

$$
\begin{aligned}
\rho\left[\frac{\partial \mathbf{u}}{\partial t}+(\mathbf{u} \cdot \nabla) \mathbf{u}\right] & =-\nabla p+\eta \nabla^{2} \mathbf{u} \\
\nabla \cdot \mathbf{u} & =0
\end{aligned}
$$

where $\mathbf{u}$ is the velocity vector, $\rho$ is the fluid density, $\eta$ is the fluid viscosity, $t$ is the time, and $p$ is the pressure. Typically, the values for the density and the viscosity are set to those for water at room temperature, i.e. $10^{3} \mathrm{~kg} / \mathrm{m}^{3}$ and $10^{-3} \mathrm{~kg} /(\mathrm{ms})$, respectively. The equations are solved for the steady state flow for a pressure driven fluid. The boundary conditions are a $0.01 \mathrm{~m} / \mathrm{s}$ inlet velocity oriented along the longitudinal axis of the channel, zero pressure at the outlet, and no-slip at all the solid surfaces. The flow field equations are solved using a generalized minimal residual method (GMRES) iterative solver with a geometrical multigrid pre-conditioner and a Vanka algorithm for the pre- and post-smoothing. The typical number of elements in the mesh used is 60000 , which in the limit of low Reynolds numbers ensures that the element size is sufficiently small for the solution to be independent on the mesh size.

The mixing profiles of the two fluids are visualized using the solution to the convection-diffusion equation for a concentration field:

$$
\frac{\partial c}{\partial t}=D \nabla^{2} c-\mathbf{u} \cdot \nabla c
$$

where $c$ is the concentration of tracer species in the fluids, $D$ is the diffusion constant, and $\mathbf{u}$ is the vector field. The tracers are considered to be non-interacting massless points that are not affecting the flow field. Thus after solving the Navier-Stokes equations for the flow, the solution to the velocity field is saved and it is subsequently used to solve the convection-diffusion equation for the steady state. To avoid the numerical errors sometimes associated with the convection-diffusion equation, the mesh 
used was denser (up to 140000 elements). Consequently the solution for the velocity field obtained from the NavierStokes equations was mapped onto the new mesh. Also, the convection-diffusion equation was solved by employing a direct solver using Gaussian elimination, rather than an iterative one. The diffusion constant $D$ was fixed within the range of $0.5-1.2 \times 10^{-9} \mathrm{~m}^{2} / \mathrm{s}$, which is the corresponding diffusion range for most ions in aqueous solutions. The solution to the diffusion equation is evaluated in the steady flow state with discontinuous concentration profiles across the inlet, generated using an Heaviside step function smoothed to the second order.

To quantify the mixing quality we are using an entropic measure applied to the tracer species concentration images obtained at different positions along the channel length. The entropy is the unique and universal mixing measure $[32,33]$ that depends only on the tracer probability distribution. We evaluate the quality of mixing of two fluids carrying two types of tracers. The space of interest is divided into $M$ equal sized regions labeled $j=1,2, \ldots M$, and there are two species of tracers $t=1,2$. The overall entropy is given by:

$$
S=-\sum_{t=1}^{2} \sum_{j=1}^{M} p_{t, j} \ln p_{t, j}
$$

where $p_{t, j}$, the joint probability for a tracer to be of species $t$ and in region $j$, is estimated by the fraction of tracers of species $t$ located in region $j$ out of all tracers. Following [34-36], the following probabilities are used to express the entropy $S$ as a sum of conditional entropies:

$$
S=S(\text { locations })+S_{\text {locations }}(\text { species })
$$

where:

$$
\begin{aligned}
S(\text { locations }) & =-\sum_{j=1}^{M} p_{j} \ln p_{j} \\
S_{\text {locations }}(\text { species }) & =\sum_{j=1}^{M} p_{j} S_{j}(\text { species }) \\
S_{j}(\text { species }) & =-\sum_{t=1}^{2} p_{t / j} \ln p_{t / j},
\end{aligned}
$$

$S$ (locations) is the entropy associated with the overall spatial distribution of tracers irrespective of species. Since the fluid is incompressible, $S$ (locations) does not change along the length of the mixer. For this reason, we concentrate on the second term on the right hand side of equation (6), i.e. $S_{\text {locations }}$ (species) which is an average of the $S_{j}$ (species) entropy of mixing of tracers species conditional on location. Since $0 \leqslant S_{\text {locations }}($ species $) \leqslant \ln (2)$, we get can define the mixing index as: $S_{\text {locations }}($ species $) / \ln (2)$. Thus the mixing index has its lowest value 0 when for all the locations the $p_{t / j}$ probabilities are either zero or one (complete segregation), and it has its largest value 1 when all probabilities are equal to each other (complete mixing). In all the results reported here the entropy was computed using $M=30351$ bins.

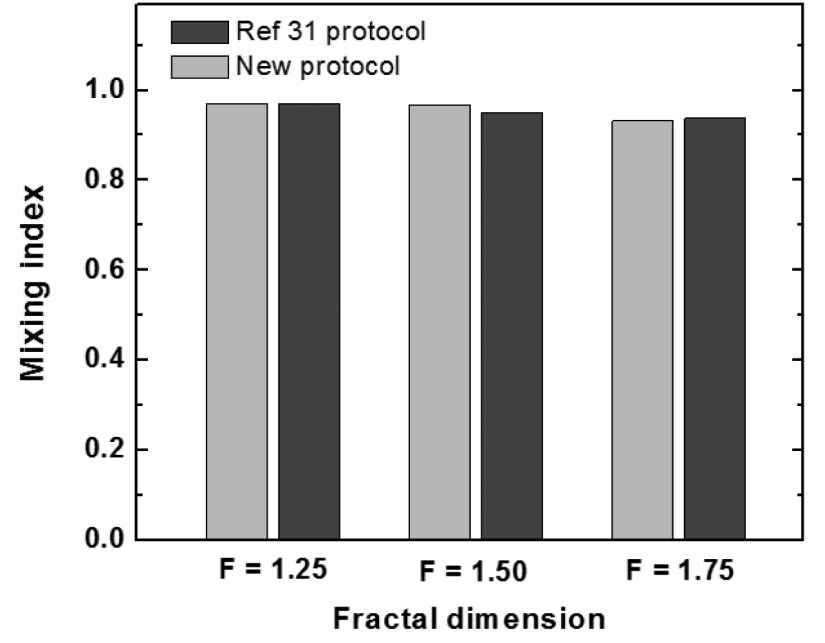

Fig. 3. Comparison between mixing in different fractal dimension mixers generated using reference [31] protocol and the new protocol described in the text. $(F=1.25,1.5$ and 1.75 respectively.)

\section{Results and discussion}

While the first computational analysis of fractal surface patterning induced flows [31], has demonstrated the potential for these designs to be superior in terms of mixing quality to geometrically similar SHB channels, no attempt has been made to optimize their performance for geometrical parameters achievable through replica molding based lithography. For example, the width of the ridges considered was far smaller than the approximately $\sim 10 \mu \mathrm{m}$ achievable through soft lithography without costly photomasks. While in our modelling and optimization we have used experimentally feasible widths for the ridges and as described in Section 2 above a new protocol for the structure design, the new geometries still show similar improvements in the value of the mixing indices (Fig. 3).

In this study, we have chosen to focus on the geometrical optimization of the micromixers with respect to two geometrical parameters: the spacing between the ridges and the range of the $y$-position of their apex. For all the designs the ridge height and width are fixed at $50 \mu \mathrm{m}$. Similar with computational results on the SHB the performance of our mixers is dependent on both these geometrical parameters (Fig. 4). For a narrow spacing between the ridges the mixing is poor because the amount of fluid entering the grooves between the ridges and gaining a non-axial component to the velocity is relatively small compared with the total fluid volume transmitted through the channel. In the other extreme case, i.e. very large ridge spacing, the number of instances at which the flow field is disturbed from the global axial direction is greatly reduced, leading to an increase of the distance required for efficient mixing. Regarding the apex range, a maximum is observed for an intermediate range between the two extremes considered. In the extreme case in which the apex of all the ridges is fixed along the median of the channel bottom, even in the presence of the two counter rotating flows, due to the symmetry of the problem the fluid mixing is still limited 

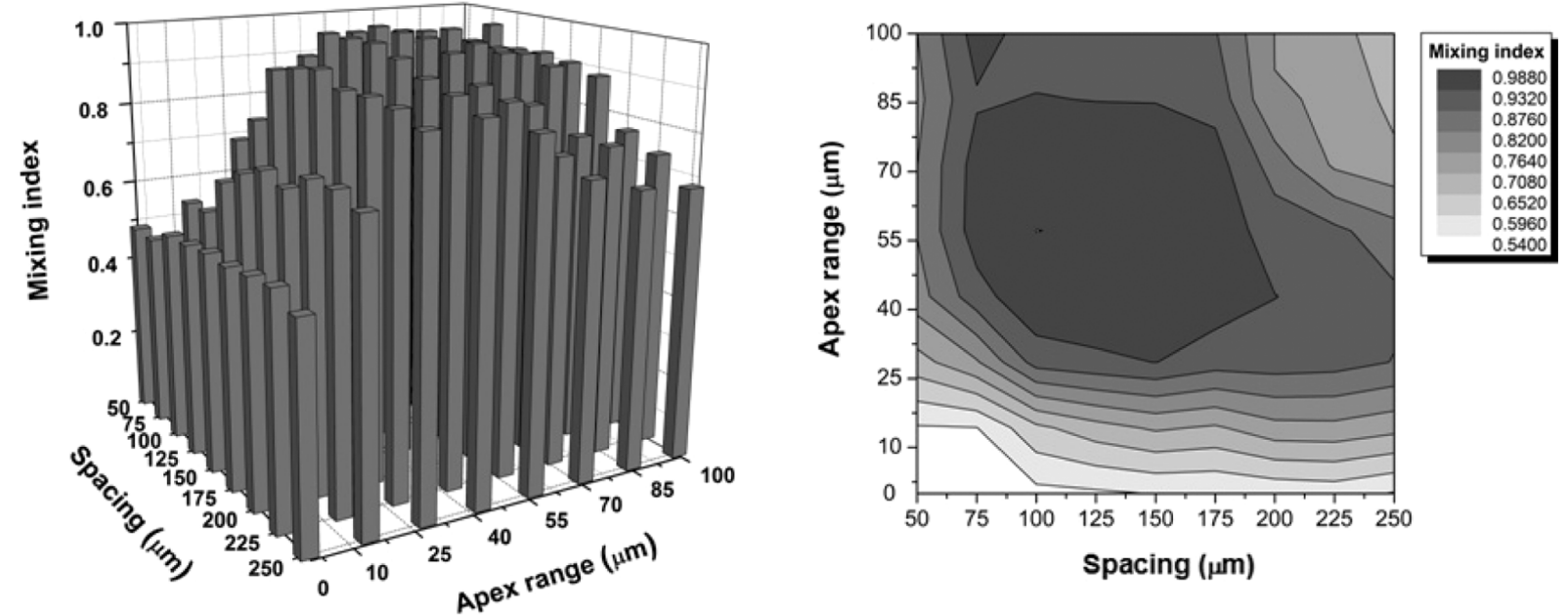

Fig. 4. Mixing index as function of groove spacing and apex position range for channels with patterns of fractal dimension $F=1.25$. The spacing is changed in increments of $25 \mu \mathrm{m}$ from 50 to $250 \mu \mathrm{m}$, while the limits of the apex position range are increased in steps of $15 \mu \mathrm{m}$.

to the initial interface between the two fluids. If the ridge apex is allowed to span the entire width of the channel, for a sizable number of ridges the apex is too close to the sidewalls of the channel to induce comparable counter rotating flows. In this extreme case, the design resembles the less efficient slanted groove micromixer one $[37,38]$. To optimize the design and fully map the mixing index, the spacing between the ridges is changed from $50 \mu \mathrm{m}$ to $250 \mu \mathrm{m}$ in $25 \mu \mathrm{m}$ steps, while the apex range is adjusted from the full width of the channel $(=200 \mu \mathrm{m})$ to the extreme in which all the ridge tips are aligned along the longitudinal symmetry axis of the channel. The global maximum for the mixing measure is obtained for a spacing between the ridges, corresponding to $125 \mu \mathrm{m}$ and an apex range of $\pm 55 \mu \mathrm{m}$ around the symmetry axis of the channel.

After identifying the optimal geometrical parameters for the fractal based designs, three geometries with fractal dimension $F=1.25,1.5$, and 1.75 , were compared with the benchmark provided by SHB geometry. For the fractal designs, the ridge apex range is fixed at $\pm 55 \mu \mathrm{m}$, while for the SHB the optimal $2 / 3$ ratio [30] (i.e. the apexes $y$-coordinate is either $-66 \mu \mathrm{m}$ or $+66 \mu \mathrm{m})$ is used. For the SHB designs, the number of grooves per half-period changes from 3 to 9 as the spacing between the ridges is decreased from $250 \mu \mathrm{m}$ to $50 \mu \mathrm{m}$. Calculations of the mixing index at the outlet, using the entropic measure, indicate that for all the spacing values considered the fractal designs show consistently better mixing quality than the SHB counterparts for all the fractal dimensions used (Fig. 5). One important feature of the fractal based designs is the broader set of spacing values at which optimal mixing is achieved when compared with the SHB design. This statement can be debated based on the fact that the fractal designs require a larger set of different values for the groove apex positions that have to be experimentally implemented with good spatial resolution during the fabrication step of the channels. Nevertheless, numerical

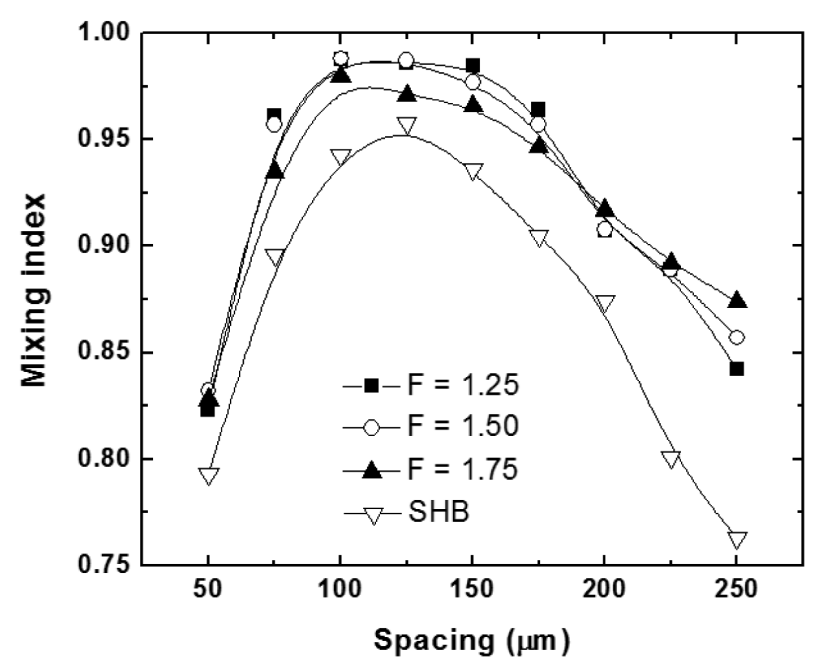

Fig. 5. Mixing dependence at the outlet of the microchannels for optimized $F=1.25, F=1.5, F=1.75$ (apex range $\pm 55 \mu \mathrm{m}$, spacing $125 \mu \mathrm{m}$ ) and SHB designs.

results show that even if an experimental resolution limit of $10 \mu \mathrm{m}$ is imposed on these designs, their mixing efficiency remains practically unchanged. From the point of view of the actual experimental implementation of this protocol, this could help relax the requirements on the resolution of the soft lithography fabrication process. In particular during the replica molding step some stretching of the PDMS elastomer can occur distorting the features transferred from the master.

A qualitative analysis of the dynamics of the mixing from tracer images at different cross sections along the channels (Fig. 6), reveals that similarly with the case of SHB designs the mixing is driven by the helical flows generated as the fluid is pressure driven over the $\mathrm{V}$-shaped grooves and ridges. The apparent blurring of the interface between different tracer species is a consequence of the inclusion in the convection-diffusion equation of finite 


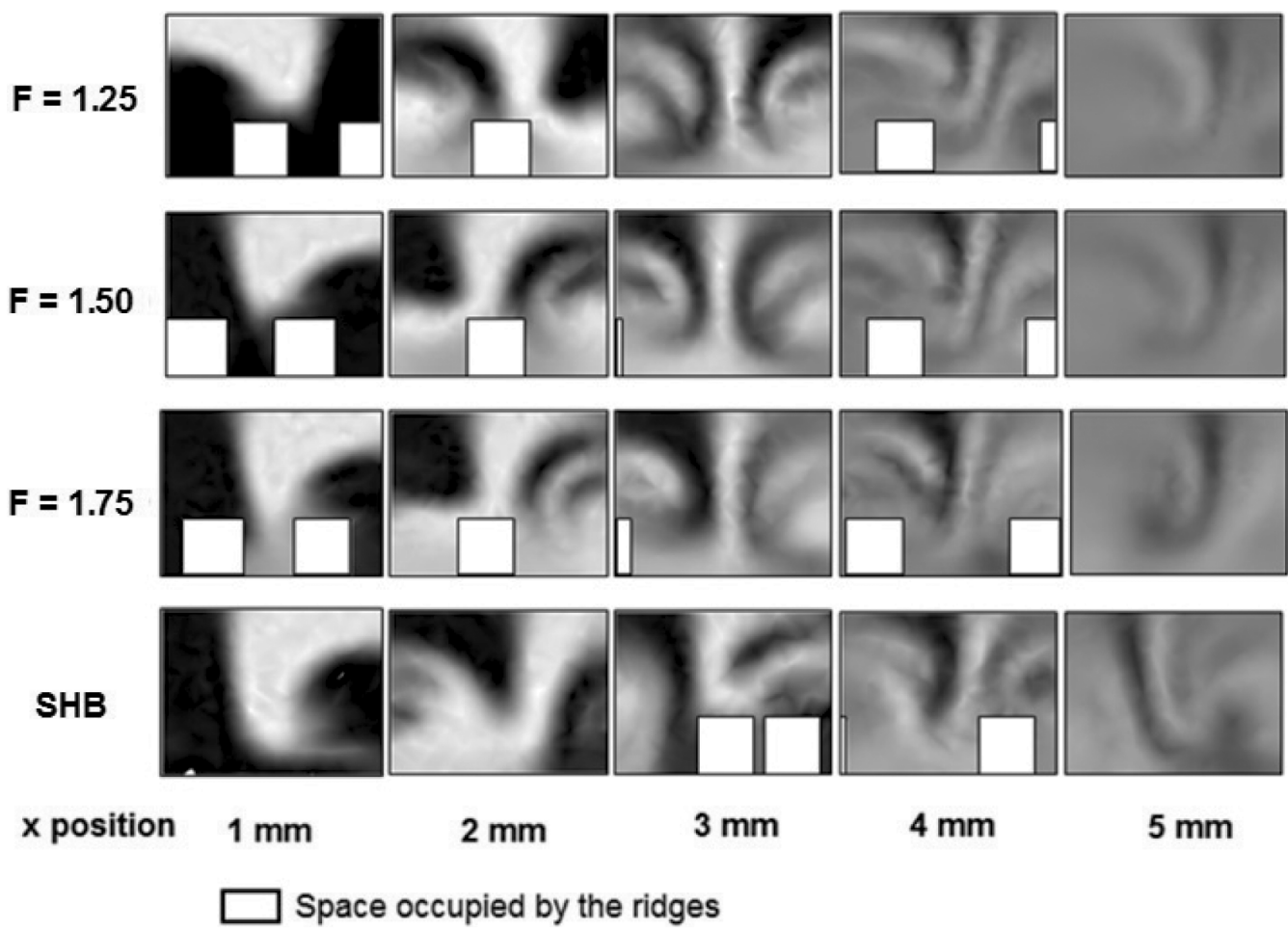

Fig. 6. Snapshots of the mixing at different positions on the channel longitudinal axis for $F=1.25,1.5,1.75$ and $\mathrm{SHB}$ geometries.

values for the diffusion constant. Evaluating the mixing efficiency, for different diffusion coefficients but similar flow fields can allow the decoupling, in the evaluation of the mixing quality of the geometrically driven convection from the diffusion effects. For all the designs investigated the contribution of the diffusion to the mixing is estimated to be less than $10 \%$ by evaluating the mixing for different diffusion constant $D$ values. This value also corresponds to the mixing value obtained through an identical computational procedure, in benchmark rectangular channels without surface patterning in which the mixing is only diffusive in nature. The Reynolds number for most of the systems investigated is: $R e=\rho v L / \eta=$ $\left(10^{3} \mathrm{~kg} / \mathrm{m}^{3}\right)\left(10^{-2} \mathrm{~m} / \mathrm{s}\right)\left(10^{-4} \mathrm{~m}\right) /\left(10^{-3} \mathrm{~kg} /(\mathrm{ms})\right)=1$. This value implies that the flow is laminar. On the other hand, the Peclet number, is $P e=v L / D \sim 10^{3}$, for diffusion constants $D$ of the order of $10^{-9} \mathrm{~m}^{2} / \mathrm{s}$. Thus the mixing observed in this study is primarily due to transversal flows induced by nonlinearities in the boundary conditions, i.e. ridges on the walls, and is not due to turbulence or diffusion.

While it is relatively easy to conclude from the tracer maps the presence of transversal flows within all the patterned channels investigated, no fundamental difference is observed between the nature of these flows in the SHB designs and the fractal pattern ones. The better mixing quality in the later is probably related with a system that is more complex due to the non-periodic boundary conditions imposed. However, in this study no attempt has been made to quantify the chaotic character of the flow fields. Nevertheless, the enhancement in the mixing quality is also observable in the dependence of the mixing index along the channel (Fig. 7). Past the first ridge, both for the fractal design with $F=1.25$ as well as for the SHB geometry the mixing index increases almost monotonically along the channel length. The small non-zero value for the evaluation of the mixing index at the beginning of the channel is related with the smoothed step function used to generate the tracer concentration profile at the inlet. The maximum mixing achieved within the length of the channels is $98 \%$ for the fractal design and $95 \%$ for the SHB one. For the SHB channel the mixing length (the length over which $90 \%$ mixing is achieved) is estimated to be about $0.45 \mathrm{~cm}$. This value corresponds well with previously reported values $[20,25]$, with the slight discrepancy being due to the finite value for the diffusion constant used in our study. For exactly the same geometrical parameters and fluid properties, the $F=1.25$ fractal design achieves the same mixing quality within a length of about $0.4 \mathrm{~cm}$. This is also qualitatively observed in tracer concentration image slices (Fig. 7b) along the length of the channel which show a faster homogenization for the $F=1.25$ geometry.

\section{Conclusions}

We have performed an extensive computational assessment of the mixing efficiency of rectangular microchannels in which a fractal function protocol is used to create a complex set of ridges on the channel floor. The quality of 
P.S. Fodor et al.: Passive micromixers with fractal surface patterning

(a)


(b)

Fig. 7. (a) Calculated dependence of the mixing index on the $x$-position along the channel. (b) Top-down view along the channel for SHB and $F=1.25$ geometries.

mixing, calculated using an entropic measure, is found to be sensitive to geometrical parameters such as the spacing between the ridges and the allowed range for their apex. While the dependence on the geometrical parameters and the flow fields is qualitatively similar between the fractal channels and the SHB channels, a better mixing is achieved for the former ones. We also find that the range of the geometrical parameters for which optimal performance is achieved is broader for the non-periodic designs. This is a desirable feature as it relaxes the demands on the fabrication process. For all the microchannels explored, the geometrical parameters used were chosen within the range accessible to soft lithography techniques. Despite their more complicated geometry, for the experimental implementation of the designs proposed, the only overhead, when compared with other similar microchannels, would occur during the computer aided design (CAD) of the photolithography mask. Nevertheless, the script and data import features of the majority of CAD packages will allow this overhead to be reduced to a minimum. While all the fractal dimensions used showed the capability to promote enhanced mixing, no attempt has been made in this study to quantify a dependence on it. Future work will focus on microchannels with a sufficiently large numbers of ridges to allow an analysis of the fractal character of the fluid mixing. Another interesting problem is the dispersion of advected particles due to stress forces imparted by the fluid. This was studied analytically [39] for a rectangular channel without ridges and we plan to extend that work to the flow conditions of the fractal channels studied here.

This work was supported through a research grant from the Cleveland State University Provost office.

\section{References}

1. G.M. Whitesides, Nature 442, 368 (2006)

2. L. Kang, B.J. Chung, R. Langer, A. Khademhosseini, Drug Discovery Today 13, 1 (2008)

3. R. Daw, J. Finkelstein, Nature 442, 367 (2006)

4. R.E. Oosterbroek, A. Bergn, Lab on a Chip: Miniaturized Systems for (bio)chemical Analysis and Synthesis (Elsevier, Amsterdam, 2006)

5. R. Barry, D.J. Ivanov, J. Nanobiotechnol. 2, 2 (2004)

6. P.S. Dittrich, A. Manz, Nature Rev. Drug Discovery 5, 210 (2006)

7. R. Ehrnström, Lab. Chip 2, 26N (2002)

8. J.C. McDonald, D.C. Duffy, J.R. Anderson, D.T. Chiu, H. Wu, J.A. Shuller, G.M. Whitesides, Electrophoresis 21, 27 (2000)

9. H.A. Stone, A.D. Stroock, A. Ajdari, Annu. Rev. Fluid Mech. 36, 381 (2004) 
10. T.M. Squires, S.R. Quake, Rev. Mod. Phys. 77, 977 (2005)

11. N.S. Lynn, C.S. Henry, D.S. Dandy, Microfluid. Nanofluid. 5, 493 (2008)

12. H. Suzuki, C.M. Ho, N. Kasagi, J. Micromech. Syst. 13, 779 (2004)

13. K. Horiuchi, P. Dutta, C.D. Richards, Microfluid. Nanofluid. 3, 347 (2007)

14. Z. Yang, H. Goto, M. Matsumoto, R. Maeda, Microelectromech. Syst. 80 (2000)

15. D. Therriault, S.R. White, J.A. Lewis, Nat. Mat. 2, 265 (2002)

16. J. Cha, J. Kim, S.K. Ryu, J. Park, Y. Jeong, S. Park, S. Park, H.C. Kim, K. Chun, J. Micromech. Microeng. 16, 1778 (2006)

17. J.M. Park, D.S. Kim, T.G. Kang, T.H. Kwon, Microfluid. Nanofluid. 4, 513 (2008)

18. A.P. Sudarsan, V.M. Ugaz, Lab. Chip 6, 74 (2006)

19. F. Jiang, K.S. Drese, S. Hardt, M. Kupper, F. Schonfeld, AIChE J. 50, 2297 (2004)

20. A.D. Stroock, S.K. Dertinger, A. Ajdari, I. Mezic, H.A. Stone, G.M. Whitesides, Science 295, 647 (2002)

21. D.S. Kim, S.W. Lee, T.H. Kwon, S.S. Lee, J. Micromech. Microeng. 14, 798 (2004)

22. H. Sato, S. Ito, K. Tajima, N. Orimoto, S. Shogi, Sens. Actuat. A Phys. 119, 365 (2005)

23. D.G. Hassell, W.B. Zimmerman, Chem. Eng. Sci. 61, 2977 (2006)

24. S.P. Kee, A. Gavriilidis, Chem. Eng. Sci. 142, 109 (2008)

25. C.A. Li, T.N. Chen, Sens. Actuat. B 106, 871 (2005)
26. M.S. Williams, K.J. Longmuir, P. Yager, Lab. Chip 8, 1121 (2008)

27. H.Z. Wang, P. Iovenitti, E. Harvey, S. Masood, J. Micromech. Microeng. 13, 801 (2003)

28. J. Aubin, D.H. Fletcher, C. Xuereb, Chem. Eng. Sci. 60, $2503(2005)$

29. N.S. Lynn, D.S. Dandy, Lab. Chip 7, 580 (2007)

30. J.T. Yang, K.J. Huang, Y.C. Lin, Lab. Chip 5, 1140 (2005)

31. M. Camesasca, M. Kaufman, I. Manas-Zloczower, J. Micromech. Microeng. 16, 2298 (2006)

32. C.E. Shannon, W. Weaver, Mathematical Theory of Communication (University of Illinois Press, Urbana, 1963)

33. A.I. Khinchin, Mathematical Foundations of Information Theory (Dover, New York, 1957)

34. M. Camesasca, I. Manas-Zloczower, M. Kaufman, J. Micromech. Microeng. 15, 2038 (2005)

35. M. Camesasca, M. Kaufman, I. Manas-Zloczower, Macromol. Theory Simul. 15, 595 (2006)

36. M. Kaufman, M. Camesasca, I. Manas-Zloczower, L.A. Dudik, C. Liu, in Functionalized Nanoscale Materials, Devices and Systems (Springer, The Netherlands, 2008), p. 437

37. T.J. Johnson, D. Ross, L.E. Locascio, Anal. Chem. 74, 45 (2002)

38. A.D. Stroock, S.K. Dertinger, G.M. Whitesides, A. Ajdari, Anal. Chem. 74, 5306 (2002)

39. M. Kaufman, Nanoscale Microscale Thermophys. Eng. 11, $129(2007)$

To access this journal online: www.edpsciences.org 